\title{
Increased Risk of Cerebral Thrombo-embolic Complication during Gradual Carotid Artery Occlusion Combined with Extra- and Intracranial Bypass Surgery in the Treatment of an Inaccessible Carotid Aneurysm
}

\author{
Toru Hayakawa, Yoshikazu Iwata, Kazuo Yamada, Norio ARita, \\ Shintaro MoRı, Tadahisa MızuTA and Yukitaka UsHIo
}

Department of Neurosurgery, Osaka University Medical School, Osaka

\begin{abstract}
We report a 31-year-old woman with an intracavernous carotid aneurysm who experienced a cerebral embolic complication during the gradual occlusion of an internal carotid artery (ICA) following superficial temporal to middle cerebral artery anastomosis. The cerebral angiogram revealed an embolus lodged in the stem of the middle cerebral artery. Based on our findings we suggest that in the presence of a sufficient collateral blood supply after extra- and intracranial bypass surgery, abrupt ICA ligation may reduce the risk for thrombo-embolic complications. If, on the other hand, the collateral blood flow is insufficient, gradual ICA occlusion should be considered and anticoagulant therapy should be used.
\end{abstract}

Key words: carotid artery, cerebral aneurysm, cerebral embolism and thrombosis, cerebral ischemia, cerebral revascularization, postoperative complication

\section{Introduction}

Cervical carotid artery ligation is effective in the treatment of intracavernous and/or giant carotid aneurysms. However, immediate or delayed cerebral ischemic complications represent a serious problem. ${ }^{9,11)}$

To prevent this complication, gradual, rather than abrupt, closure of the carotid artery has been recommended $^{4,9)}$ and extra- and intracranial (EC-IC) bypass surgery has been performed. ${ }^{3,7)}$ Spetzler et $a .^{12)}$ suggested that the combination of both procedures, i.e., gradual occlusion of the internal carotid artery (ICA) and EC-IC bypass surgery, may be a safer treatment.

We encountered cerebral thrombo-embolic complications in a patient with an intracavernous carotid aneurysm who was treated by gradual occlusion of the ICA after anastomosis of the superficial temporal
(STA) and middle cerebral arteries (MCA).

\section{Case Report}

The patient was a 31-year-old woman. Cerebral angiographic examination, performed prior to surgery for prolactinoma, disclosed an aneurysm of the right ICA just proximal to the origin of the ophthalmic artery (Fig 1). On March 3, 1982, the pituitary adenoma was successfully removed via the sublabial rhinoseptal transsphenoidal approach.

On March 23, 1982, a Selverstone clamp was applied to the right cervical ICA and right STA-MCA anastomosis with double branches was performed. Changes in the regional cortical blood flow (rCBF) were determined intraoperatively by the heat clearance method, using two probes placed on the surface of the right frontal and temporal lobes at craniotomy. While the anastomosed STA was closed

Received February 27, 1984; Accepted June 11, 1984

Author's present address: Y. Iwata, M.D. and T. Mizuta, M.D., Department of Neurosurgery, Minoo City Hospital, Minoo, Osaka, Japan; S. Mori, M.D., Department of Neurosurgery, The Center for Adult Diseases, Osaka, Japan. 


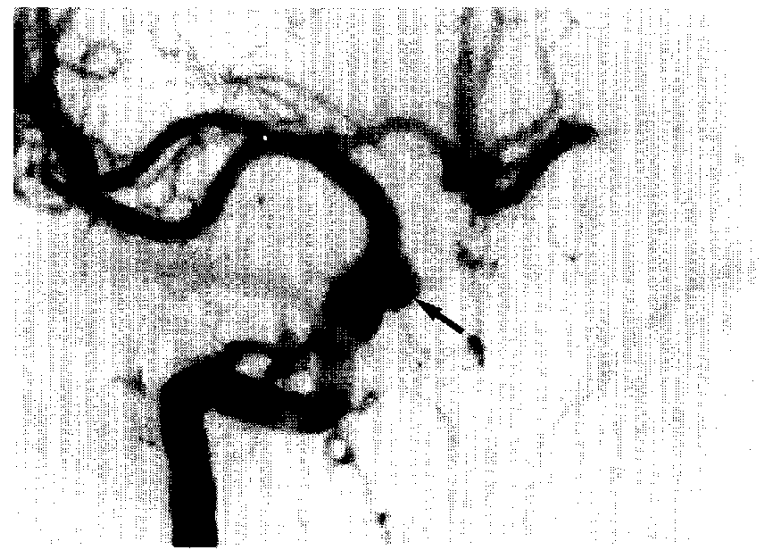

Fig. 1 Preoperative right carotid angiogram (A-P view) showing an aneurysm of the right internal carotid artery (arrow).

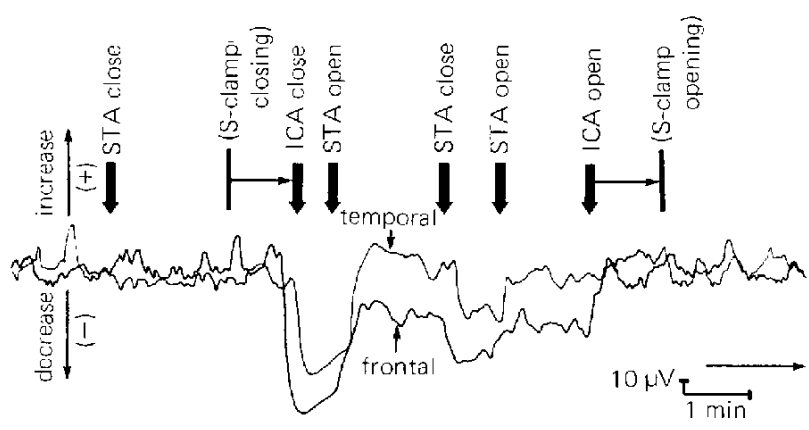

Fig. 2 Changes in regional cortical blood flow levels measured by the heat clearance method. While the anastomosed STA was closed with a clip, the flow levels of both the frontal and temporal lobes decreased upon closure of the right cervical internal carotid artery; these levels recovered, although not completely, upon opening the anastomosed STA. S-clamp: Selverstone clamp.

by a clip, $\mathrm{rCBF}$ in the frontal and temporal lobes decreased upon closure of the right cervical ICA with the clamp (Fig. 2). The decrease in rCBF was canceled, although not completely, by opening the anastomosed STA. This observation suggested that the rCBF of the right hemisphere was dependent on the bypass collaterals, when the right ICA was occluded and that the bypass flow did not suffice to compensate completely for the decrease in $\mathrm{rCBF}$. The arterial blood pressure of the right ICA, just distal to the clamp, was measured by inserting a needle, which was connected to a pressure straingauge and a polygraph. With the clamp open, the mean arterial blood pressure of the right distal ICA was 100
$\mathrm{mmHg}$. After closing the clamp, the stump pressure in the ICA dropped to $50 \mathrm{mmHg}$. Therefore, the Selverstone clamp was partially opened so that the blood pressure of the right distal ICA was maintained halfway between these two values $(75 \mathrm{mmHg})$. The vessel was probably occluded by about $75 \%$. Postoperatively, the patient was alert and showed no neurological deficits.

On the morning of the 2nd postoperative day, the Selverstone clamp was advanced to about $80 \%$ occlusion. The patient tolerated this procedure well and no neurological changes ensued. However, early in the next morning, she noted weakness of the left upper extremity, which progressed rapidly to left hemiparesis and was accompanied by dysarthria (Fig. 3). A CT scan revealed no apparent abnormality and the cerebral angiogram showed that the right ICA was completely occluded at the site of the clamp and that the anastomoses were patent. There was cross-collateral flow from the left ICA to the right anterior and middle cerebral arteries through the anterior communicating artery. Embolic stenosis was detected in the stem of the right MCA at the origin of the lenticulostriate arteries (Fig. 4).

The Selverstone clamp was completely closed and volume expansion and mild hypertension therapy were performed thereafter. Starting that evening, her hemiparesis and dysarthria gradually improved. A CT scan performed the next day revealed a small lowdensity area in the right caudo-putaminal region and the internal capsule.

After physical therapy, the patient was discharged with mild weakness of the left upper extremity.

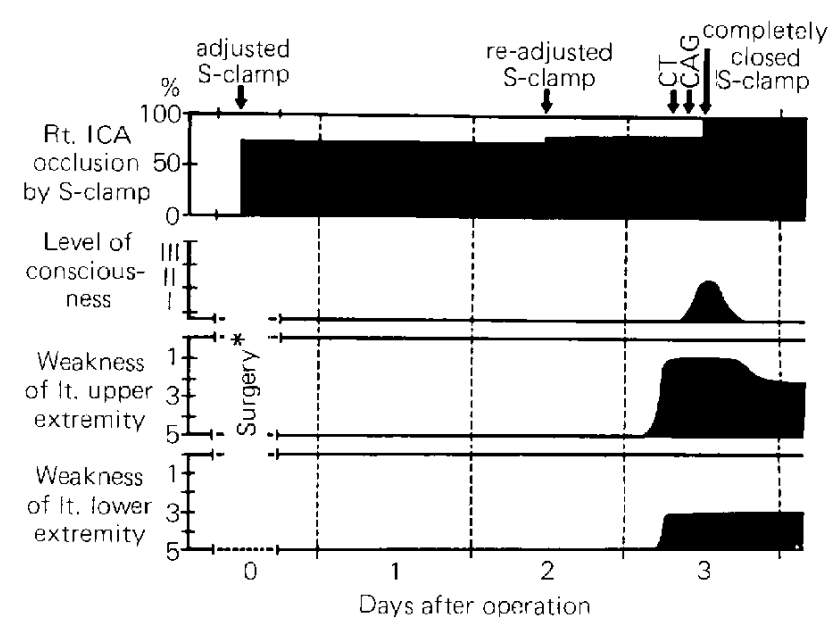

Fig. 3 Schematic illustration of the clinical course. *Right STA-MCA anastomosis and Selverstone clamp placement on right internal carotid artery.

Neurol Med Chir (Tokyo) 25, January 1985 


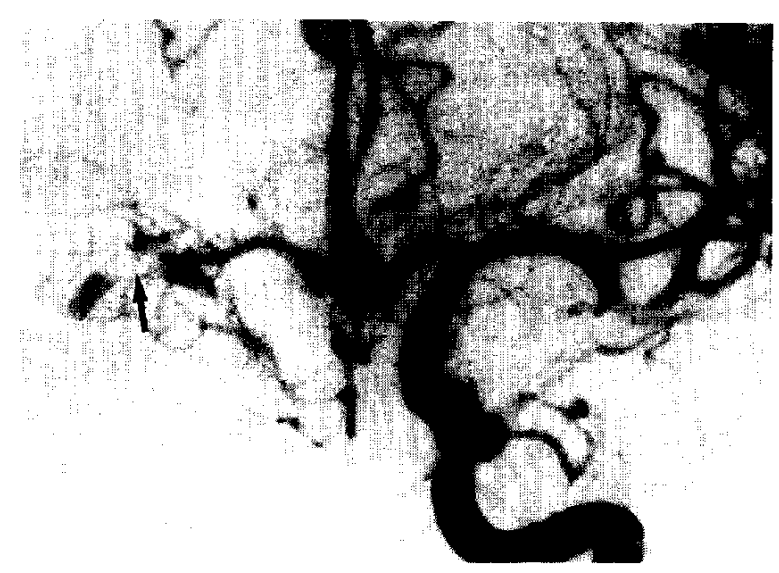

Fig. 4 Postoperative left carotid angiogram (A-P view). There is cross-collateral blood flow from the left internal carotid to the right anterior cerebral artery and to parts of the right internal carotid and middle cerebral arteries through the anterior communicating artery. Note the embolic stenosis in the stem of the right middle cerebral artery (arrow).

\section{Discussion}

Cerebral ischemia is a serious complication in the therapeutic ligation of the carotid artery, particularly the ICA, and the occurrence of ischemia may be ascribable to low blood perfusion more often than to thrombo-embolism. To avoid complications due to low perfusion, it is necessary to assess the collateral circulation after carotid ligation pre- and intraoperatively by, for example, the EEG Matas test, angiographical assessment of cross filling, stump pressure measurement of the carotid artery, and rCBF study. In our patient, we found $\mathrm{rCBF}$ measurement, using the heat clearance method, useful.

To aid in the development of a collateral blood supply, gradual occlusion of the carotid artery has been employed, ${ }^{4,9)}$ and after the establishment of the microsurgical technique, EC-IC bypass surgery is frequently combined before carotid ligation. ${ }^{3,7}$ Spetzler et $a l .^{12)}$ have recommended combined therapy of the gradual occlusion of the ICA and EC-IC bypass surgery as a safer treatment. However, in some patients subjected to this combined therapy, embolic complications have occurred. ${ }^{2,6)}$ In our patient, the presence of a patent anastomosis and findings by CT scanning and cerebral angiography suggest that the ischemic symptom may be attributable to the lodging of an embolus in the stem of the right MCA. This embolus may have obstructed the right lenticulostriate arteries, although the low perfusion due to thrombic occlusion of the right ICA may have played a role.

The mechanism(s) underlying the increased risk of embolic complications after this combined treatment remains obscure. The embolus may originate in the aneurysmal cavity ${ }^{(0)}$ or the poststenotic part of the partially clamped ICA. There is blood flow turbulence at both sites, which may promote thrombus formation. Furthermore, jet flow in the poststenotic region of the $\mathrm{ICA}^{1,8)}$ may accelerate the separation of the embolus from the thrombic lesion. To date, embolization has not been emphasized as a cause of ischemic complications during or after therapeutic gradual carotid occlusion. ${ }^{6}$ In most reports, the neurological disorders occurring during or after gradual ICA occlusion have only been described as ischemic complications and their etiology, in the absence of angiographic documentation, has not been specified.

What role does combined EC-IC bypass surgery play in the occurrence of postoperative ischemic complications? It is reasonable to postulate that combined EC-IC bypass surgery in patients subjected to gradual ICA occlusion may enhance the relative stasis of blood flow and may lead to turbulence in the ipsilateral distal ICA, MCA and aneurysmal sac. This may accelerate thrombus formation and thus, increase the risk of embolic complications. STA-MCA anastomosis sometimes produces to and fro flow in the stem of the ipsilateral MCA (watershed area) and a floating micro-embolus may lodge in the vessel wall in this watershed area. Thereafter, it may increase in size and result in an ischemic lesion and some neurological deficits. Figure 5 is a schematic illustration of this hypothetical mechanism.
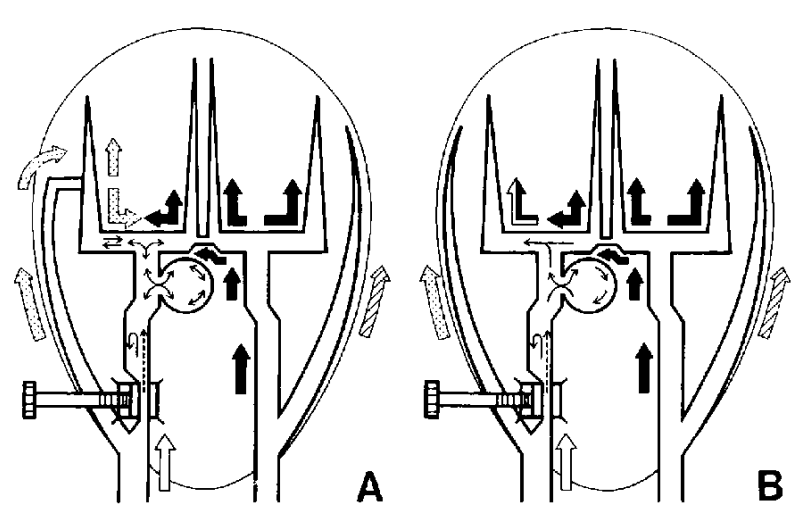

Fig. 5 Schematic illustration of blood flow turbulence in the presence of an aneurysm, stenosis of the internal carotid artery by application of a Selverstone clamp with (A) or without STAMCA anastomosis (B). 
Currently, the gradual occlusion of the ICA combined with EC-IC bypass surgery is popular in the treatment of ICA aneurysms inaccessible to direct surgery. However, our experience and increasing evidence $^{2,6)}$ reported by others indicate that the gradual occlusion of the ICA presents the risk of thrombo-embolic complications, and combined bypass surgery may increase this risk. Recently, Hashi ${ }^{5)}$ reported, based on the statistical analysis of the reported cases, that there were three risk factors for ischemic complications in the therapeutic carotid ligation and they consisted of advanced age, left ICA ligation and also gradual occlusion of the ICA. Available evidence would indicate that easy selection of the combined therapy of gradual occlusion of the ICA and bypass surgery from the viewpoint of preventing complications due to low perfusion may not be safe. We agree with Heros et al. ${ }^{6}$ and would suggest that in the presence of a sufficient collateral blood supply after bypass surgery, abrupt ligation of the ICA should be performed and it may reduce the risk for thrombo-embolic complications. On the other hand, when the collateral blood flow appears to be insufficient, like in the reported case, that gradual occlusion of the ICA might be considered but, in the case, anticoagulant therapy, suggested by Diaz et $a l .{ }^{2)}$ should be combined for preventing thrombo-embolic complications.

\section{Conclusions}

Gradual occlusion of carotid artery using Selverstone clamp may not be safe for carotid artery ligation in the treatment of an inaccessible carotid aneurysm, especially when combined with EC-IC bypass surgery. In the presence of a sufficient collateral blood supply, abrupt ICA ligation would be recommended.

\section{Acknowledgments}

We are grateful to Professor H. Mogami, Department of Neurosurgery, Osaka University Medical School, for his advice and guidance for this study.

Part of this work was presented at the 41 th Annual Meeting of the Japanese Neurosurgical Society, October, 1982, Tokyo.

\section{References}

1) Blackshear WM Jr, Phillips D, Chikos PM, Harley JD, Thiele BL, Strandness DE Jr: Carotid artery velocity patterns in normal and stenotic vessels. Stroke 11: 67-71, 1980

2) Diaz FG, Ausman JI, Pearce JE: Ischemic complications after combined internal carotid artery occlusion and extracranial-intracranial anastomosis. Neurosurgery 10: 563-569, 1982

3) Gelber BR, Sundt TM Jr: Treatment of intracavernous and giant carotid aneurysms by combined internal carotid ligation and extra- to intracranial bypass. $J$ Neurosurg 52: 1-10, 1980

4) Giannotta SL, McGillicuddy JE, Kindt GW: Gradual carotid artery occlusion in the treatment of inaccessible internal carotid artery aneurysms. Neurosurgery 5: 417-421, 1979

5) Hashi K: Pitfalls \& complications of neurosurgical procedures. Carotid ligation. Presented at 4th Annual Meeting of the Japanese Congress of Neurological Surgeons. Osaka, 1984

6) Heros RC, Nelson PB, Ojemann RG, Crawell RM, DeBrun G: Large and giant paraclinoid aneurysms: Surgical techniques, complications, and results. Neurosurgery 12: 153-163, 1983

7) Hopkins LN, Grand W: Extracranial-intracranial arterial bypass in the treatment of aneurysms of the carotid and middle cerebral arteries. Neurosurgery 5: 21-31, 1979

8) Moritake $\mathrm{K}$, Handa $\boldsymbol{H}$, Yonekawa $\mathrm{Y}$, Takebe $\mathrm{Y}$, Kishimoto S, Makimoto K: Ultrasonic Doppler assessment of hemodynamics in gradual carotid ligation. Stroke 12: 177-178, 1981

9) Nishioka $\mathrm{H}$ : Report on the cooperative study of intracranial aneurysms and subarachnoid hemorrhage. Section III, Part 1. Results of treatment of intracranial aneurysm by occlusion of the carotid artery in the neck. I Neurosurg 25: 660-682, 1966

10) Pozzati E, Fagioli L, Servadei F, Gaist G: Effect of common carotid ligation on giant aneurysms of the internal carotid artery: Computerized tomographic study. J Neurosurg 55: 527-531, 1981

11) Roski RA, Spetzler RF, Nulsen FE: Late complications of carotid ligation in the treatment of intracranial aneurysms. J Neurosurg 54: 583-587, 1981

12) Spetzler RF, Schuster H, Roski RA: Elective extracranial-intracranial bypass in the treatment of inoperable giant aneurysms of the internal carotid artery. $J$ Neurosurg 53: 22-27, 1980

Address reprint requests to: T. Hayakawa, M.D., Department of Neurosurgery, Osaka University Medical School, 1-1-50 Fukushima, Fukushima-ku, Osaka 553 , Japan. 